\title{
Length of stay, mortality, and readmissions among Medicare cancer patients treated with glucarpidase and conventional care: a retrospective study
}

This article was published in the following Dove Medical Press journal: ClinicoEconomics and Outcomes Research

\author{
Berna Demiralp' \\ Lane Koenig' \\ Jaya Kala ${ }^{2}$ \\ Chaoling Feng' \\ Elizabeth G Hamlett ${ }^{1}$ \\ Marie Steele-Adjognon' \\ Suzanne Ward ${ }^{3}$ \\ 'KNG Health Consulting, Rockville, \\ MD, USA; ${ }^{2}$ University of Texas \\ Health Science Center, University of \\ Texas MD Anderson Cancer Center, \\ Houston, TX, USA; ${ }^{3}$ BTG International, \\ West Conshohocken, PA, USA
}

Correspondence: Berna Demiralp KNG Health Consulting, 15245 Shady Grove Road - Suite 365, Rockville, MD 20850, USA

Tel +I 2404030154 ext 303

Fax + I 3013398436

Email berna.demiralp@knghealth.com
Purpose: Glucarpidase (Voraxaze) is used to treat methotrexate (Mtx) toxicity in patients with delayed Mtx clearance due to impaired renal function. We examine hospital length of stay (LOS), mortality, and readmission rates for Medicare cancer patients with delayed clearance of Mtx treated with glucarpidase.

Methods: Using 2010-2017 Medicare claims data, we identified glucarpidase patients as those hospitalized with indications of select lymphomas or leukemia, inpatient chemotherapy, and glucarpidase treatment. We assessed outcomes of glucarpidase patients relative to those experienced by patients treated for presumed Mtx toxicity using other therapies. These nonglucarpidase patients were identified with a diagnosis of primary central nervous system lymphoma, indications of cancer-chemotherapy toxicity, and acute kidney injury during hospitalization (not present on admission), and were divided into two groups: treated with dialysis (dialysis ${ }^{+}$) and treated with or without dialysis (dialysis ${ }^{+-}$). Inverse-probability treatment weighting using propensity scores was used to adjust for differences between groups.

Results: Patients treated with glucarpidase $(n=30)$ had an average LOS of 14.7 days. They had inpatient, 30-day, and 90-day mortality rates of 3.3\%, $13.3 \%$, and $16.7 \%$, respectively, and a 90-day all-cause unplanned readmission rate of $24.1 \%$. The dialysis ${ }^{+}$and dialysis ${ }^{+/}$groups, respectively, had higher average LOS $(40.2,21.9)$, higher inpatient mortality $(50.6 \%, 20.8 \%)$, and higher 90 -day mortality $(58.6 \%, 37.6 \%)$. No statistically significant differences in 30-day mortality or 90-day readmission rates were detected between the glucarpidase group and either of the nonglucarpidase groups. Unobservable differences in patient severity may impact the interpretation of our findings.

Conclusion: Medicare cancer patients with presumed Mtx toxicity receiving conventional treatment experience long hospitalizations, high intensive-care unit use and high mortality. Glucarpidase patients had lower LOS, inpatient mortality, and 90-day mortality than the nonglucarpidase patients.

Keywords: chemotherapy, toxicity, methotrexate, outcomes

\section{Introduction}

Glucarpidase (Voraxaze) is used to treat methotrexate (Mtx) toxicity in patients with delayed Mtx clearance due to impaired renal function. In this study, we examined hospital length of stay (LOS), mortality, and readmission rates among Medicare beneficiaries with cancer treated with and without glucarpidase. Nonglucarpidase patients were those treated for presumed Mtx toxicity using other therapies based on 
clinical indicators observed in the Medicare claims data. We also conducted an exploratory analysis, comparing outcomes between patients who received glucarpidase within 3 days of admission to the hospital and those who received it after 3 days of admission.

\section{Literature review}

We conducted a systematic literature review on the effectiveness of glucarpidase in treating Mtx toxicity. The Supplementary material includes our literature review methodology and attrition chart. Of the 28 studies included in the literature review, 6 were observational, ${ }^{1-6} 5$ were literature reviews, ${ }^{7-11}$ and 17 were clinical case studies. ${ }^{12-28}$ Across all included studies, researchers assessed the impact of glucarpidase administration on patients with delayed Mtx clearance and/ or Mtx-induced toxicity. The most commonly studied patient outcomes related to our study were patient mortality and time to renal recovery. We found no studies on the impact of glucarpidase on readmission rates.

The majority of the studies identified in the literature review were clinical case studies, which examined patient mortality. Among patients included in the clinical case studies, nearly all (24 of 26) who received glucarpidase rescue survived the toxicity. ${ }^{12-28}$ Mortality was also a common outcome in the observational studies; however, there was variation across studies in terms of the period over which mortality was measured and whether overall mortality or mortality related to high-dose Mtx (HDMtx) was reported. Across included observational studies, overall mortality varied from $6.2 \%{ }^{3}$ to $44.2 \%,{ }^{6}$ and mortality directly attributed to complications due to HDMtx ranged from $0^{5}$ to $23.3 \%$. $^{6}$

Schwartz et $\mathrm{al}^{6}$ conducted an observational study of 43 adult and elderly (aged 18-78 years, median age of 54 years) acute lymphocytic leukemia, lymphoma, germ-cell tumor, and osteosarcoma patients treated with glucarpidase. The majority of patients experienced grade III-IV toxicities, including hematotoxicity, mucositis, nephrotoxicity, and hepatotoxicity. All patients received glucarpidase, followed by leucovorin and standard supportive care. Ten patients died of HDMtx-associated complications, including infection, uremia, neurotoxicity, and peritonitis with multiorgan failure. Unlike Schwartz et al, ${ }^{6}$ both Widemann et $\mathrm{al}^{4}$ and Buchen et $\mathrm{al}^{3}$ observed glucarpidase rescue among younger cancer patients. Buchen et al performed a prospective, open, nonrandomized, multicenter trial for 65 patients suffering from acute lymphocytic leukemia, non-Hodgkin's lymphoma, osteosarcoma, and various other cancers. Patients received 33-60 U of glucarpidase $/ \mathrm{kg}$ body weight, followed by leucovorin and standard supportive care.
Despite glucarpidase rescue, three patients died due to severe myelosuppression and sepsis related to Mtx toxicity. Widemann et $\mathrm{al}^{4}$ conducted a retrospective study of 100 cancer patients to assess the impact of glucarpidase, leucovorin, and thymidine on patients suffering from Mtx toxicity and reduced renal function. Glucarpidase was administered at a median of 96 hours post-Mtx administration if alongside thymidine and 66 hours post-Mtx administration if alone. A total of 12 patients died, half attributed to delayed glucarpidase administration and irreversible Mtx toxicity. In their 2014 retrospective study, Widemann et $\mathrm{al}^{9}$ assessed the effectiveness of glucarpidase rescue for patients experiencing renal toxicity and delayed elimination of Mtx. Of the 492 patients included in the study, $8 \%$ died within 30 days of glucarpidase administration. However, all deaths were ruled unrelated to glucarpidase by the patient's treating physician. In Flombaum et al's ${ }^{2}$ observational study on seven cancer patients treated with glucarpidase, one patient expired due to complications related to sepsis. Additional research on the impact of the time of glucarpidase administration was conducted by Ward et al, ${ }^{1}$ who concluded that increased patient mortality was associated with delayed administration.

Our examination of prior literature reviews yielded three additional observational studies that reported mortality among patients treated with glucarpidase. Widemann et $\mathrm{al}^{9}$ examined mortality among 20 osteosarcoma and gastric cancer patients receiving glucarpidase. Two patients expired, one from HDMtx-related myelosuppressive and pulmonary complications. In their literature review, Cavone et al reported on Krause et al's study, in which the authors examined eight adult cancer patients experiencing renal impairment and delayed Mtx clearance, and all patients survived following treatment with glucarpidase. ${ }^{11}$ Fermiano et al's literature review discusses Christensen et al's study, in which the authors focused on 20 pediatric patients suffering from Mtx induced acute kidney injury (AKI). ${ }^{10}$ All patients received glucarpidase and supportive care, and survived treatment.

Although we identified no studies that explicitly reported LOS, several studies reported time to renal recovery after Mtx-induced toxicity. Among the clinical case studies that reported this outcome, time to renal recovery ranged from 7 days post-HDMtx administration ${ }^{17}$ to 6 weeks, ${ }^{13}$ with the majority of studies reporting time to renal recovery of $<1$ month. Among the observational studies included in the literature review, median time to renal recovery ranged from 12.5 days post-glucarpidase administration ${ }^{5}$ to 22 days post-HDMtx administration. ${ }^{4}$ In their 2004 literature review of clinical case studies about osteosarcoma patients using HDMtx, Widemann et $\mathrm{al}^{9}$ reported that patients treated with 
supportive-care measures, including dialysis, resumed "normal" renal function within a median of 16 days, and patients treated with glucarpidase resumed "normal" renal function within a median of 22 days.

Our literature review revealed several gaps in the literature. First, the literature lacks studies that directly compare outcomes between patients who received and did not receive glucarpidase. Second, the studies we found focused on a limited set of patient outcomes that included mortality and time to renal function. We aim to fill these gaps in the literature with our study. Specifically, we contribute to prior literature by assessing patient outcomes associated with glucarpidase relative to outcomes experienced by similar patients who are not treated by glucarpidase. In addition, we examine episodebased patient outcomes and resource utilization associated with glucarpidase. To our knowledge, this study is the first claims-based analysis on the outcomes of patients treated with glucarpidase.

\section{Immunosuppressive agent and toxicity}

Mtx is an anticancer and immunosuppressive agent that is used in the treatment of rheumatoid arthritis, psoriasis, and various malignancies. It is an antifolate that prevents the synthesis of folic acid, thereby inhibiting cell growth. HDMtx, administered intravenously at doses of $500 \mathrm{mg} / \mathrm{m}^{2}$ or higher, is frequently used to treat lymphoblastic leukemia, primary central nervous system lymphoma (PCNSL), and other lymphomas. ${ }^{29}$ It can cause significant toxicity and renal dysfunction, including renal failure and death. ${ }^{6,8,29-31}$

Mtx toxicity refers to toxic plasma Mtx concentrations $>10 \mu \mathrm{mol} / \mathrm{L} 24$ hours after administration, $>1 \mu \mathrm{mol} / \mathrm{L} 48$ hours after administration, or $>0.1 \mu \mathrm{mol} / \mathrm{L} 72$ hours after administration. ${ }^{32} \mathrm{AKI}$ results from crystal nephropathy, a process in which Mtx precipitates within the renal tubules. This crystalinduced nephropathy begins with an asymptomatic elevation in serum creatinine levels, and can develop into tubular necrosis and AKI. ${ }^{29}$ This impairment can lead to delayed excretion of Mtx through the kidneys and induce other toxicities and adverse effects. ${ }^{29}$ Prolonged kidney dysfunction results in continued accumulation of Mtx at toxic levels, and this with increased systemic Mtx exposure can cause other toxic reactions, including myelosuppression, mucositis, hepatotoxicity, and in severe cases multiorgan failure. ${ }^{29}$

In general, $\mathrm{AKI}$ is considered an important predisposing factor to end-stage renal disease (ESRD) and a risk factor for other outcomes, such as recurrent hospitalizations, future AKI, cardiovascular events, and mortality. ${ }^{33} \mathrm{~A}$ recent publication revealed that patients who survive an AKI episode have double the risk of death, triple the risk of ESRD, and ten times the risk of developing incident or progressive chronic kidney disease (CKD).$^{33}$ The rate of cardiovascular events is as high as $22 \%$, and mortality related to cardiovascular events is $33 \%$ in patients with AKI, and even higher in those with a history of diabetes mellitus, hypertension, prior cardiovascular diseases, and those who develop CKD. ${ }^{34}$ Though some AKI episodes may be considered harmless, poor cardiovascular outcomes indicate the presence of systemic damage secondary to the inflammatory process during the AKI episode. This may continue even after the patient has survived the initial AKI episode and has regained baseline renal function. ${ }^{34}$ Although the extent to which HDMtx-induced AKI follows patterns observed in the general AKI population is unknown, the poor outcomes observed in the general AKI population raises concerns about the potential adverse effects of HDMtx-induced AKI.

Due to the potentially harmful effects of Mtx toxicity on renal function, patients treated with HDMtx are closely monitored and provided supportive care, including hydration, urinary alkalinization, and leucovorin (folinic acid). ${ }^{29,35}$ Patients exhibiting toxic plasma Mtx levels and delayed elimination during treatment receive aggressive supportive care consisting of high doses of leucovorin and/or frequent doses of intravenous fluids. ${ }^{3,35}$ As the patient shows signs of renal insufficiency and reduced Mtx clearance, glucarpidase and/ or dialysis is added to the supportive-care treatment regimen to accelerate Mtx clearance. ${ }^{6,9,17,36}$ The resulting reductions in plasma Mtx allow leucovorin to work more effectively and counter the effects of Mtx intracellularly. Although glucarpidase is typically administered once the patient demonstrates acute kidney dysfunction, some clinicians suggest it should be administered earlier, when the patient first begins exhibiting symptoms of AKI and elevated plasma Mtx levels. ${ }^{6,36}$

\section{Clinical strategies}

Vigorous hydration and urinary alkalinization are considered standards of care to be administered prior to and during Mtx therapy. Because Mtx is eliminated through the kidneys, the use of fluids can enhance normal Mtx elimination by promoting high urinary flow rates and alkalinizing the urine, which prevents crystallization of Mtx. These processes protect the kidneys from injury and mitigate the onset of other toxicities. Many pediatric protocols recommend at least 2 hours of hyperhydration with a minimum of $200 \mathrm{~mL} / \mathrm{m}^{2}$ per hour or $100-150 \mathrm{~mL} / \mathrm{m}^{2}$ per hour beginning 12 hours before the start of Mtx infusion and continuing for 24-48 hours or longer if the patient is at risk of Mtx toxicity or presents signs of 
delayed Mtx elimination. Similar protocols are commonly indicated for adults, including administration of bicarbonatecontaining fluids at rates of $150-200 \mathrm{~mL}$ per hour to a total of $2 \mathrm{~L}$ prior to HDMtx infusion and urine alkalinization with a target urine $\mathrm{pH}>7 .{ }^{29}$

Leucovorin has been in use as a rescue therapy for HDMtx toxicity for $>30$ years. It is considered effective in the prevention of specific toxicities associated with HDMtx treatment, including myelosuppression, gastrointestinal toxicity, and neurotoxicity. While some have found leucovorin to be sufficient in treating HDMtx toxicity, others have argued that at high Mtx concentrations $(>100 \mu \mathrm{M})$, leucovorin may not be as effective, leaving the patient vulnerable to kidney injury and other associated toxicities. ${ }^{29,37,38}$

Dialysis involves the removal of Mtx from the blood through diffusion. Two main types of dialysis, hemodialysis and peritoneal, are most commonly used for patients experiencing HDMtx-induced toxicity and related kidney injury. ${ }^{29}$ However, dialysis is usually ineffective in the removal of Mtx, except for high-flux hemodialysis, which may not be readily available or suitable for patients and may require multiple sessions. ${ }^{39}$

Glucarpidase is used to lower toxic Mtx levels rapidly among patients with renal impairment and delayed clearance of Mtx. It works by breaking Mtx into two inactive compounds that can be excreted nonrenally. ${ }^{8,29}$ Glucarpidase was available on a compassionate-use basis from 1993 until it was approved by the US Food and Drug Administration in January 2012. ${ }^{29,40}$ Prior literature on the effectiveness of glucarpidase, consisting primarily of case reports and clinical studies, has found it to be an effective treatment for Mtx toxicity, reducing plasma Mtx concentration by $>97 \%$ within 15 minutes of administration..$^{3,4,6,35,40-42}$ Research also suggests that early administration of glucarpidase ( $<96$ hours after start of HDMtx) may lower the risk of developing grade 4 toxicity and reduce the risk of mortality in patients who receive glucarpidase within 2 days of HDMtx. ${ }^{1,4}$ Recently developed guidelines on the use of glucarpidase recommend the administration of glucarpidase within 48-60 hours after the start of HDMtx infusion with indication of delayed Mtx clearance. $^{43}$

\section{Methods}

\section{Data and study population}

Data for the analysis came from Medicare inpatient standard analytic files (SAFs) and denominator files between Q1 2010 and Q3 2017. We used Medicare inpatient SAFs to obtain data on $100 \%$ of Medicare fee-for-service (FFS) claims for short-term acute-care hospitals. Denominator files were used to gather information on patient demographics, Medicare part A and B coverage information, and date of death. The Medicare FFS patient population was chosen as the study population due to the availability of the detailed diagnostic and inpatient hospital utilization information available in Medicare claims. While the majority of the Medicare population consists of beneficiaries aged 65 years and older, it also includes patients younger than 65 years, who are eligible for Medicare primarily due to disability.

Medicare SAF data do not permit direct identification of patients who have received Mtx or leucovorin or had Mtx toxicity. Consequently, our study population was selected based on the following criteria designed to capture patients with Mtx toxicity as accurately as possible, given the limitations of the data: 1) patients hospitalized in a short-term acute-care hospital for the treatment of cancers commonly treated with Mtx (lymphosarcoma, reticulosarcoma, other specified malignant tumors of lymphatic tissue including PCNSL, lymphoid leukemia, and other malignant lymphomas), and 2) patients who received chemotherapy during their inpatient stay. We excluded the following patients to ensure that their entire care episode was observable in claims data: 1) patients without part A coverage during their hospitalization and for 90 days following discharge, 2) patients enrolled in a Medicare Advantage plan during hospitalization or the 90 days following discharge, and 3) patients discharged against medical advice. Part A coverage refers to coverage for inpatient hospitalizations, and Medicare Advantage plans are offered by private companies approved by Medicare. Table S1 reports definitions for all variables used to define the study population. Medicare claims data used in the analysis were obtained as limited data sets under a data-use agreement, and thus did not require authorization from study subjects or documentation of a waiver according to the Privacy Rule of Health Insurance Portability and Accountability Act.

\section{Identification of glucarpidase and nonglucarpidase groups}

Cancer patients who received glucarpidase during their hospitalization based on specific ICD9 or ICD10 procedure codes were identified as the glucarpidase group (Table S1). Based on the indications of glucarpidase, we assumed that all patients treated with glucarpidase had toxic plasma Mtx concentrations.

To serve as a benchmark for the glucarpidase group's outcomes, we also constructed a nonglucarpidase group of patients who were treated for presumed Mtx toxicity using 
alternative treatments. Since Medicare claims data did not indicate whether the patient had Mtx toxicity, we identified patients for the nonglucarpidase group based on the following clinical indicators observed in the Medicare claims data. First, we identified patients who had been diagnosed with PCNSL, for which HDMtx is used as the primary inductiontreatment regimen and the most significant treatment option related to PCNSL survival. ${ }^{44-46}$ Among the PCNSL patients, we identified those who received inpatient chemotherapy and had an indication for adverse effects/poisoning due to antineoplastic and immunosuppressant drugs during their inpatient stay (Table S1). Given the relationship between Mtx toxicity and AKI, we further required that the patients did not have acute or chronic renal insufficiency present on admission but developed AKI during their hospital stay (Table S1). Elderly patients may have other comorbidities that can cause preexisting impaired renal function. By limiting the nonglucarpidase group to those who did not have prior acute or chronic renal insufficiency but developed AKI during the hospital stay, we aimed to strengthen the linkage between AKI and chemotherapy treatment. Taken together, these criteria resulted in a nonglucarpidase group that was likely to have experienced toxicity as a consequence of Mtx therapy during their inpatient stay. Finally, since all glucarpidase patients were treated in urban hospitals, we limited the nonglucarpidase group to patients in urban hospitals.

The decision to treat the patient with dialysis depends on illness severity, and is correlated with LOS, mortality and readmission rates. Consequently, we assessed the outcomes of two nonglucarpidase groups based on dialysis use. The "dialysis ${ }^{+}$" group is the subset of the nonglucarpidase group that was treated using dialysis during their inpatient stay, and the "dialysis ${ }^{+/}$" group consisted of all nonglucarpidasegroup patients, regardless of dialysis use. These patients were considered to have been given standard-of-care therapies for prevention and treatment of AKI due to HDMtx. We created the dialysis $^{+-}$group as an alternative group to conduct sensitivity analyses and assess the robustness of our findings using a broader group for comparison purposes. In addition to examining outcomes of glucarpidase patients with select lymphomas and leukemias, we also examined the outcomes of glucarpidase patients treated for PCNSL and examined their outcomes relative to those among patients in the dialysis ${ }^{+}$ and dialysis ${ }^{+/}$groups.

\section{Outcome and explanatory variables}

We assessed glucarpidase patients in terms of LOS during hospitalization, LOS in the intensive-care unit (ICU) during hospitalization, inpatient mortality, 30-day mortality after admission to hospital, 90-day mortality after admission to hospital, and 90-day postdischarge all-cause readmission rate. LOS is a proxy measure of the costs hospitals incur when treating these patients. Mortality and readmission rates are generally used as measures of clinical effectiveness. Inpatient mortality is important because an immediate treatment goal is to keep the patient alive after Mtx toxicity. Because physiological side effects caused by Mtx toxicity (eg, CKD) manifest themselves after discharge, 90-day mortality is also important. We used 30 days and 90 days as short-term and long-term goalposts, respectively, in examining mortality. Our readmission measure excluded planned readmissions, such as readmissions for maintenance chemotherapy, using an algorithm in CMS's hospital-wide all-cause readmissionmeasure definition, and was conditional on being discharged alive. $^{47}$

In comparing outcomes between the glucarpidase and the two nonglucarpidase groups, we controlled for patient age, total number of conditions in Elixhauser Comorbidity Index, congestive heart failure, cardiac arrhythmias, valvular disease, pulmonary circulation disorders, chronic pulmonary disease, complicated diabetes, renal failure (other than AKI), complicated hypertension, and tumor-lysis syndrome. ${ }^{48}$ The clinical characteristics used in risk adjustment were selected based on their importance in indicating severity and affecting outcomes in the study population. All clinical indicators were defined using diagnosis codes in the acute-care hospital, and all (other than tumor-lysis syndrome) were defined based on Elixhauser conditions.

\section{Statistical analysis}

In order to compare outcomes between the glucarpidase and nonglucarpidase groups, we conducted inverse-probability treatment weighting (IPTW) using propensity scores to control for between-group differences in patient characteristics and to make groups comparable. ${ }^{49,50}$ Specifically, we first estimated the likelihood of receiving glucarpidase within the study population using a logistic regression that controlled for patient characteristics. Based on the model estimates, we computed the predicted treatment probability, known as the propensity score, and used it to construct a patient weight that equaled the inverse of the probability of receiving/not receiving glucarpidase based on whether the patient was in the treatment or comparison group. Unadjusted outcomes were compared using $t$-tests for continuous variables and $\chi^{2}$ tests for binary variables. Adjusted differences were analyzed using Stata 14's "teffects ipw" 
command, which uses the generalized method of moments to yield consistent standard errors.

We also conducted a descriptive comparison of outcomes between patients who received glucarpidase early in their hospital stay and those who received it later. Patients who received glucarpidase within 3 days of hospital admission were identified as the early cohort and those who received it after 3 days of admission were defined as the late cohort. Assuming that Mtx infusion started on the first day of hospital admission, the use of 3 days from admission as the threshold is consistent with clinical guidelines for glucarpidase use, which recommend administering glucarpidase within 48-60 hours from the start of HDMtx infusion in patients with indication of delayed Mtx clearance. ${ }^{43}$ We compared outcomes between the two cohorts of glucarpidase patients using $t$-tests for continuous outcomes and $\chi^{2}$ tests for binary outcomes.

\section{Results}

There were 30 glucarpidase patients, 58 dialysis $^{+}$patients, and 701 dialysis $^{+/}$patients who met our inclusion/exclusion criteria (Table 1). In the glucarpidase group, 18 had lymphosarcoma, reticulosarcoma, or other specified malignant tumors of the lymphatic tissue, 2 had lymphoid leukemia, and 11 had other lymphomas, with 1 person diagnosed with both lymphoid leukemia and other lymphomas. It is important to note that although the glucarpidase group was small, it represented the entire population of Medicare FFS beneficiaries treated with glucarpidase in the USA during the study period, as indicated in Medicare claims. We note that 4 of the 30 patients in the glucarpidase group received dialysis, but the data did not allow us to evaluate whether dialysis started before or after glucarpidase had been initiated.

Descriptive statistics revealed differences between the glucarpidase and nonglucarpidase groups in terms of clinical indicators, including renal failure, complicated hypertension, tumor-lysis syndrome, and total number of Elixhauser comorbidities (Table 2). Using a standardized difference threshold of 0.2 to indicate meaningful imbalance between the two groups, we found that relative to the dialysis ${ }^{+}$group, the glucarpidase group had lower rates of pulmonary circulation disorders (3.3\% vs $8.6 \%)$ and tumor-lysis syndrome $(10.0 \%$ vs $24.1 \%$ ) and higher rates of renal failure (other than AKI; $30.0 \%$ vs $3.4 \%$ ) and complicated hypertension $(23.3 \%$ vs $3.4 \%){ }^{51,52}$ When compared to the dialysis ${ }^{+/-}$group, the glucarpidase group had higher rates of renal failure (other than AKI; 30.0\% vs $1.1 \%)$ and complicated hypertension $(23.3 \%$ vs $1.4 \%$ ) and a higher number of Elixhauser conditions, on average (2.7 vs 2.2). After IPTW, standardized differences between the glucarpidase group and nonglucarpidase groups fell below 0.20 for all of the patient characteristics examined.

\section{Hospital LOS}

Among patients who received glucarpidase, the average LOS was 14.7 days, 4.0 of which on average were spent in the ICU (Figure 1 and Tables 3 and 4). The dialysis ${ }^{+}$group had a longer average LOS (40.2), and on average patients

Table I Selection of study cohort

\begin{tabular}{|l|l|l|l|}
\hline Selection criteria & Glucarpidase $^{\text {Dialysis }}{ }^{+}$ & Dialysis $^{+-}$ \\
\hline $\begin{array}{l}\text { Had short-term acute-care hospitalization, with discharge dates between January I, 20I0 and } \\
\text { March 3I, 20I7 }\end{array}$ & $75,349,865$ & $75,349,865$ & $75,349,865$ \\
\hline Received inpatient chemotherapy during short-term acute-care hospital stay & 410,206 & 410,206 & 410,206 \\
\hline Had Part A coverage during short-term acute-care hospital stay and 90 days postdischarge & 407,32 I & 407,321 & 407,32 I \\
\hline No HMO coverage during short-term acute-care hospital stay or 90 days postdischarge & 397,716 & 397,716 & 397,716 \\
\hline Was not discharged against medical advice from the short-term acute-care hospital & 397,079 & 397,079 & 397,079 \\
\hline Hospitalized between January I, 20I0 and March 3I, 20I7 with indication of glucarpidase use & 3 I & - & - \\
\hline Diagnosed with lymphosarcoma, reticulosarcoma, lymphoid leukemia, and other lymphomas & 30 & - & - \\
\hline $\begin{array}{l}\text { Hospitalized between January I, 20I0 and March 3I, 20I7 with indication of adverse effects and } \\
\text { poisoning by antineoplastic and immunosuppressive drugs }\end{array}$ & - & 77,750 & 77,750 \\
\hline Diagnosed with central nervous system lymphoma & - & 9,562 \\
\hline No kidney injury (acute or chronic) present on admission & - & 9,562 \\
\hline Had acute kidney injury during hospital stay & - & 7,761 \\
\hline Hospitalized in an urban hospital & 30 & 722 \\
\hline Dialysis (hemodialysis or peritoneal dialysis) & 70 I & 722 \\
\hline Final cohort size & - & 58 \\
\hline
\end{tabular}

Notes: From authors' analysis of 2010-2017 Q3 Medicare inpatient standard analytic files. All claims analyses were conducted using Stata I4. 
Table 2 Patient and hospital characteristics

\begin{tabular}{|c|c|c|c|c|c|c|c|c|c|}
\hline \multirow[t]{3}{*}{ Characteristics } & \multirow[t]{3}{*}{ Glucarpidase } & \multicolumn{4}{|l|}{ Dialysis $^{+}$} & \multicolumn{4}{|l|}{ Dialysis $^{+/-}$} \\
\hline & & \multicolumn{2}{|c|}{ Unadjusted } & \multicolumn{2}{|l|}{ IPTW } & \multicolumn{2}{|c|}{ Unadjusted } & \multicolumn{2}{|l|}{ IPTW } \\
\hline & & Dialysis $^{+}$ & SD & Dialysis $^{+}$ & SD & Dialysis $^{+/-}$ & SD & Dialysis $^{+/-}$ & SD \\
\hline Sample size & 30 & 58 & - & 58 & - & 701 & - & 701 & - \\
\hline Age & 68.6 & 69.2 & -0.073 & 67.7 & 0.115 & 70.0 & -0.167 & 67.1 & 0.187 \\
\hline Age squared & $4,783.2$ & $4,838.7$ & -0.054 & $4,647.9$ & 0.131 & $4,969.8$ & -0.173 & $4,573.3$ & 0.195 \\
\hline Congestive heart failure & $13.3 \%$ & $12.1 \%$ & 0.038 & $18.8 \%$ & -0.148 & $12.0 \%$ & 0.041 & $17.5 \%$ & -0.115 \\
\hline Cardiac arrhythmias & $33.3 \%$ & $31.0 \%$ & 0.049 & $38.8 \%$ & -0.114 & $29.1 \%$ & 0.091 & $38.2 \%$ & -0.102 \\
\hline Valvular disease & $6.7 \%$ & $5.2 \%$ & 0.063 & $3.0 \%$ & 0.174 & $5.1 \%$ & 0.065 & $3.2 \%$ & 0.159 \\
\hline Pulmonary circulation disorders & $3.3 \%$ & $8.6 \%$ & -0.224 & $3.9 \%$ & -0.030 & $4.6 \%$ & -0.063 & $3.4 \%$ & -0.002 \\
\hline Chronic pulmonary disease & $13.3 \%$ & $17.2 \%$ & -0.109 & $10.3 \%$ & 0.093 & $14.0 \%$ & -0.019 & $10.7 \%$ & $0.08 \mathrm{I}$ \\
\hline Diabetes, complicated & $6.7 \%$ & $6.9 \%$ & -0.009 & $5.0 \%$ & 0.070 & $4.7 \%$ & 0.085 & $6.7 \%$ & -0.001 \\
\hline Renal failure & $30.0 \%$ & $3.4 \%$ & 0.761 & $28.6 \%$ & 0.031 & $1.1 \%$ & 0.868 & $30.4 \%$ & -0.009 \\
\hline Hypertension, complicated & $23.3 \%$ & $3.4 \%$ & 0.611 & $18.9 \%$ & 0.108 & $1.4 \%$ & 0.705 & $21.4 \%$ & 0.046 \\
\hline Total Elixhauser conditions & 2.7 & 2.6 & 0.087 & 2.7 & -0.028 & 2.2 & 0.519 & 2.7 & 0 \\
\hline Tumor lysis syndrome & $10.0 \%$ & $24.1 \%$ & -0.383 & $8.0 \%$ & 0.068 & $8.4 \%$ & 0.055 & $13.1 \%$ & -0.097 \\
\hline
\end{tabular}

Notes: From authors' analysis of 2010-2017 Q3 Medicare inpatient standard analytic files. All claims analyses were conducted using Stata 14.

Abbreviations: IPTW, inverse-probability treatment weighting; SD, standardized difference.

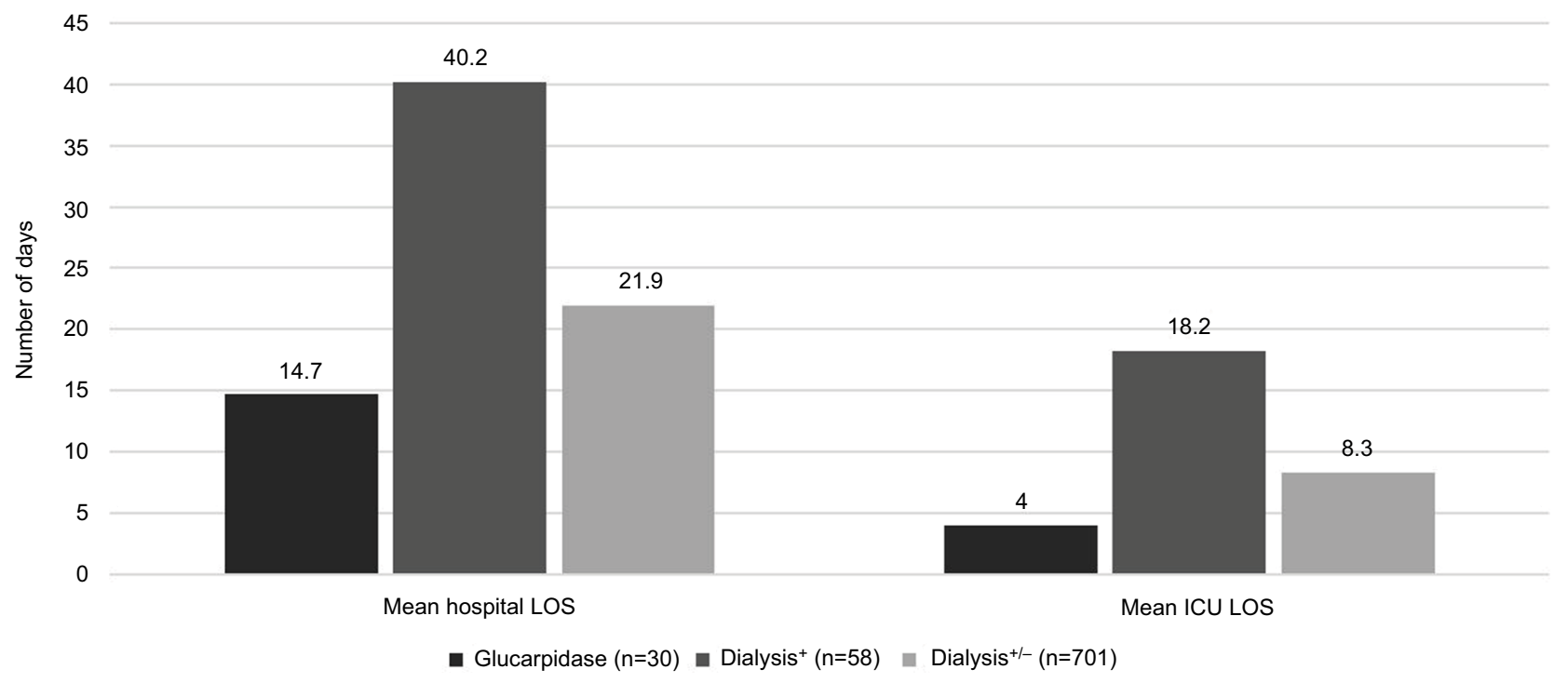

Figure I Mean length of stay (LOS) among patients treated with glucarpidase and the nonglucarpidase groups.

Notes: There was a -25.5 day difference in mean hospital LOS between the glucarpidase and dialysis ${ }^{+}$groups $(P<0.0 \mathrm{I})$ and a -7.2 day difference between the glucarpidase and dialysis ${ }^{+1-}$ groups $(P<0.05)$. There was a -14.2 day difference in mean intensive-care unit $(I C U)$ LOS between the glucarpidase and dialysis ${ }^{+}$group $(P<0.00 I)$ and a -4.2 day difference between the glucarpidase and dialysis ${ }^{+/-}$groups $(P>0.10)$. Authors' analysis of 2010-2017 Q3 Medicare inpatient standard analytic files. All claims analyses were conducted using Stata 14.

spent more days in the ICU (18.2) (Figure 1 and Table 3). Patients in the dialysis ${ }^{+/}$group spent an average 21.9 days in the hospital and 8.3 days in the ICU (Figure 1 and Table 4).

\section{Mortality and readmission}

Inpatient mortality among glucarpidase patients was $3.3 \%$ and 30 -day and 90 -day mortality $13.3 \%$ and $16.7 \%$, respectively. The dialysis $^{+}$group had a higher inpatient-mortality rate $(50.6 \%)$ and 90-day mortality rate (58.6\%; Figure 2 and Table 3 ). The dialysis $^{+-}$group had an inpatient-mortality rate of $20.8 \%$ and a 90-day mortality rate of $37.6 \%$ (Figure 2 and Table 4). We did not find a statistically significant difference between the glucarpidase group and either of the nonglucarpidase groups in terms of 30-day mortality or 90-day readmission rate. 

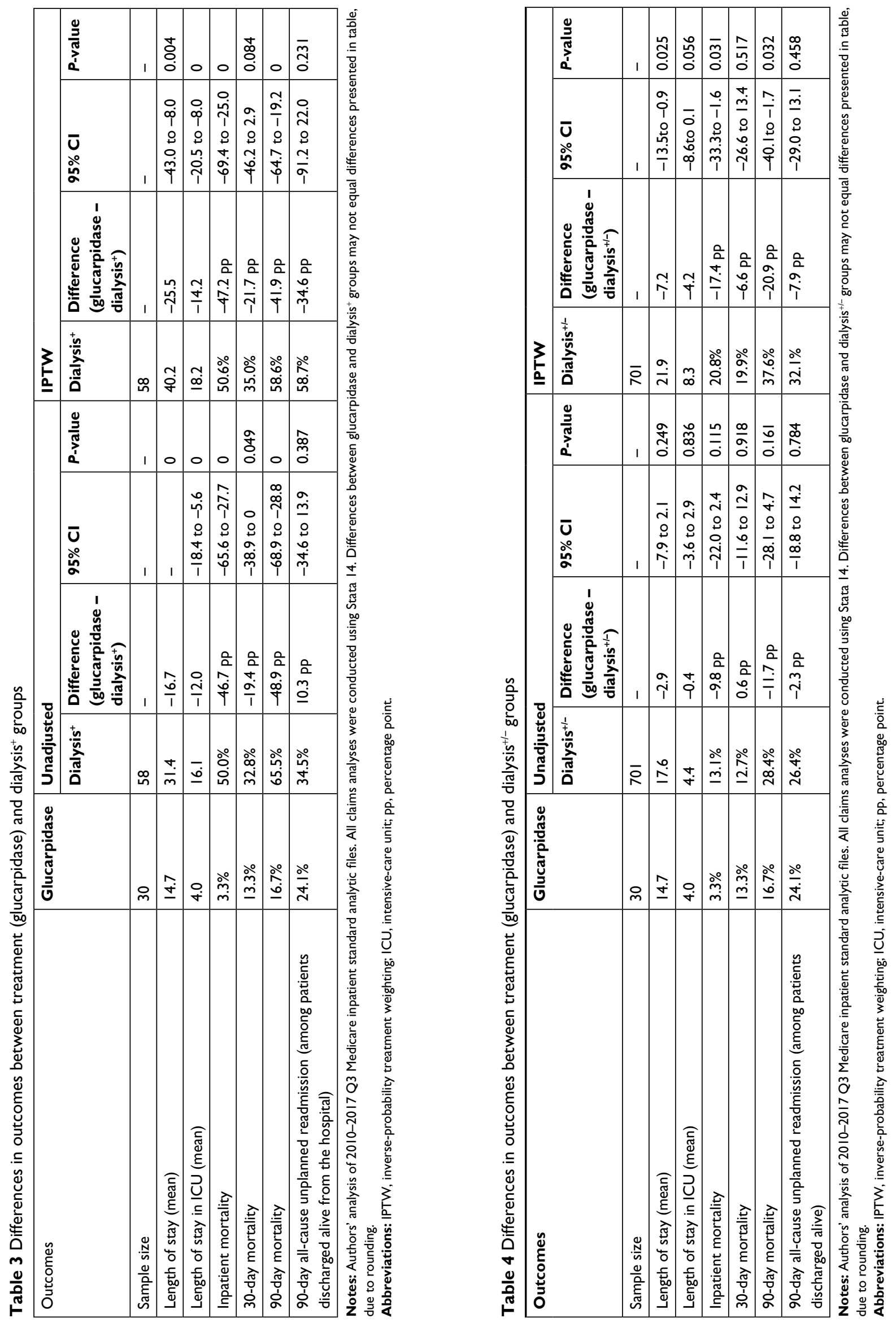


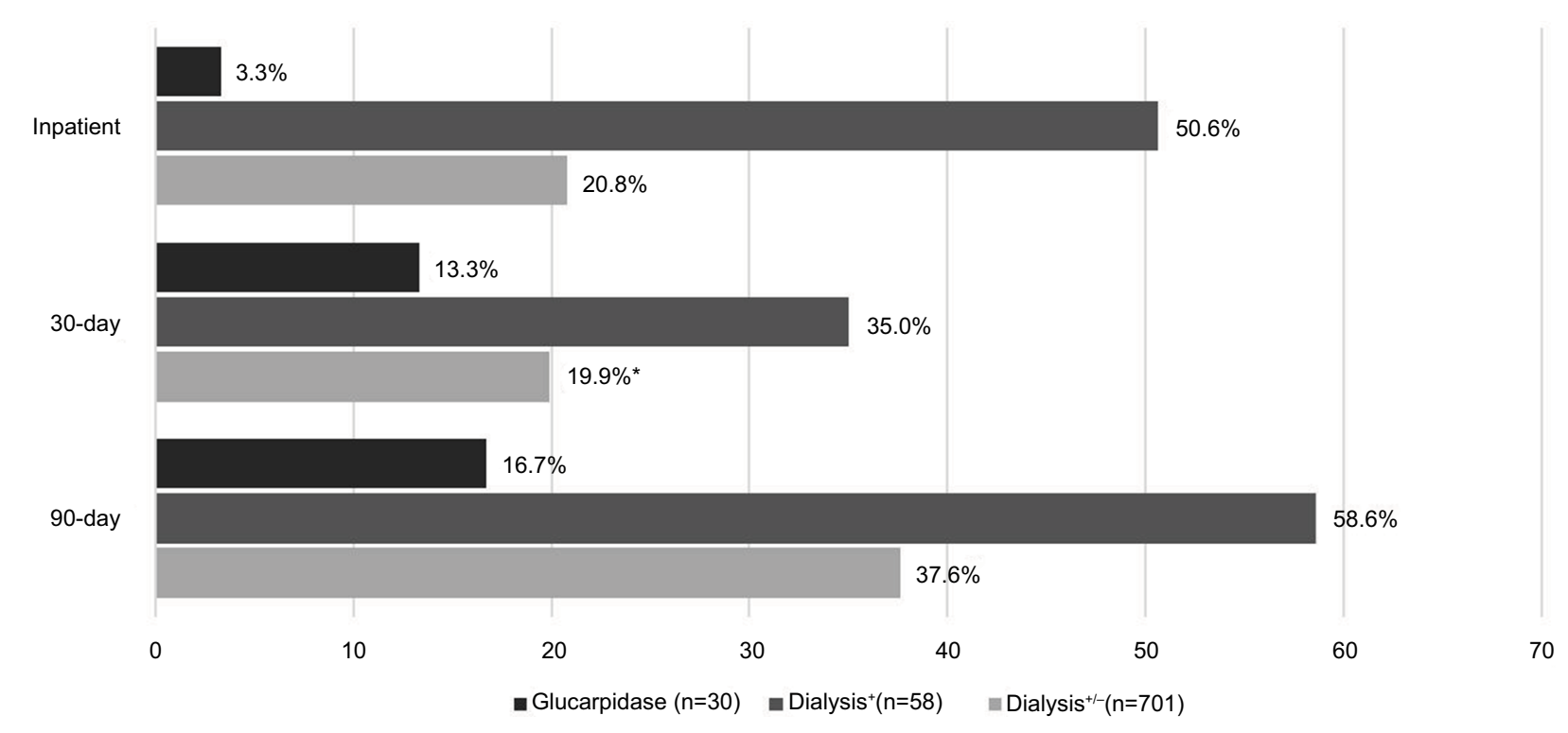

Figure 2 Rates of mortality among patients treated with glucarpidase and nonglucarpidase groups.

Notes: There was a -47.2 PP difference in inpatient mortality between the glucarpidase and dialysis ${ }^{+}$groups $(P<0.001)$; and a -17.4 PP difference between the glucarpidase and dialysis ${ }^{+-}$groups $(P<0.05)$. There was a 21.7 PP difference in 30 -day mortality between the glucarpidase and dialysis ${ }^{+}$groups $(P<0.10)$. There was a -41.9 PP difference in 90-day mortality between the glucarpidase and dialysis ${ }^{+}$groups $(P<0.001)$; and a -20.9 Pp difference between the glucarpidase and dialysis ${ }^{+-}$groups $(P<0.05)$. ${ }^{*}$ Differences in 30-day mortality rates between the glucarpidase and dialysis ${ }^{+-}$groups not statistically significant at the $10 \%$ level. Authors' analysis of $2010-2017$ Q3 Medicare inpatient standard analytic files. All claims analyses were conducted using Stata 14.

\section{Additional analyses}

We compared LOS, mortality, and readmission rates between patients who received glucarpidase within 3 days of admission to hospital and other glucarpidase patients. On average, Medicare beneficiaries in the glucarpidase group received glucarpidase within 3.7 days of admission, with $80 \%$ receiving it within 5 days of admission. Glucarpidase patients who received treatment within 3 days of admission spent on average 10.0 days in hospital and 0.8 days in the ICU (Table 5). By comparison, glucarpidase patients who received treatment after 3 days of admission spent 21.7 days on average in hospital and 8.9 days in the ICU. Average LOS following glucarpidase administration was 8.5 days in the early-treated cohort and 14.6 days in the late-treated cohort.

We also examined outcomes for glucarpidase patients diagnosed with PCNSL to examine the robustness of our findings for PCNSL patients. When the glucarpidase group was limited to the 17 cases with PCNSL, average outcomes were similar to those observed in the broader glucarpidase group. With respect to the dialysis ${ }^{+}$group, glucarpidase patients with PCNSL had lower average LOS in hospital, LOS in ICU, inpatient mortality, and 90-day mortality, with differences in outcomes between the two groups similar to those between the broader glucarpidase group and dialysis ${ }^{+}$group. With respect to the dialysis ${ }^{+/}$group, glucarpidase patients with PCNSL had lower inpatient mortality. Differences in the remaining outcomes between glucarpidase patients with PCNSL and dialysis ${ }^{+/}$patients were directionally similar but statistically insignificant at the $10 \%$ level.

\section{Discussion}

Used in the treatment of PCNSL and other cancers, Mtx can cause significant toxicity and renal impairment. Patients who are likely to be treated for Mtx toxicity using dialysis or other supportive therapies experience long hospitalizations and high mortality rates. We found that Medicare beneficiaries who are diagnosed with PCNSL and treated for chemotherapy toxicity spend on average 22 days in hospital and 8 days in the ICU, and more than a third of them do not survive 90 days from admission to hospital. Among these patients, those treated using dialysis have even longer average LOS (40 days in hospital, 18 days in ICU) and higher 90-day mortality (59\%).

Glucarpidase provides an alternative to conventional care in treating Mtx toxicity. We found that Medicare beneficiaries with select lymphomas and leukemias treated with glucarpidase had an average length of 15 days in hospital and four days in the ICU and a 90 -day mortality of $17 \%$. Although prior studies have focused on different patient populations in terms of age and cancer type, the mortality rate of our glucarpidase group was within the range of mortality rates reported in the studies identified in our literature review. An examination of 
Table 5 Comparison of outcomes between early and late cohorts of glucarpidase administration (unadjusted)

\begin{tabular}{|c|c|c|c|c|}
\hline Outcomes & $\begin{array}{l}\text { Early cohort } \\
\text { (glucarpidase date - } \\
\text { admission date } \leq 3 \text { ) }\end{array}$ & $\begin{array}{l}\text { Late cohort } \\
\text { (glucarpidase date - } \\
\text { admission date }>3 \text { ) }\end{array}$ & $\begin{array}{l}\text { Difference } \\
\text { (early - late) }\end{array}$ & P-value \\
\hline Sample size & 18 & 12 & - & - \\
\hline Length of stay & 10.0 & 21.7 & -11.7 & 0.002 \\
\hline Length of stay after glucarpidase administration & 8.5 & 14.6 & -6.1 & 0.057 \\
\hline Length of stay in ICU & 0.8 & 8.9 & -8.1 & 0.020 \\
\hline inpatient mortality & 0 & $8.3 \%$ & $-8.3 \mathrm{PP}$ & 0.213 \\
\hline 30-day mortality & $11.1 \%$ & $16.7 \%$ & $-5.6 \mathrm{PP}$ & 0.661 \\
\hline 90-day mortality & $16.7 \%$ & $16.7 \%$ & 0 & 1.000 \\
\hline $\begin{array}{l}\text { 90-day all-cause unplanned readmission (among patients } \\
\text { discharged alive from hospital) }\end{array}$ & $27.8 \%$ & $18.2 \%$ & II.I pP & 0.481 \\
\hline
\end{tabular}

Notes: Authors' analysis of 2010-2017 Q3 Medicare inpatient standard analytic files. All claims analyses were conducted using Stata 14.

Abbreviations: ICU, intensive-care unit; PP, percentage point.

outcomes between glucarpidase patients treated for select lymphomas and leukemias and PCNSL patients likely treated for Mtx toxicity revealed that glucarpidase patients had shorter LOS, lower inpatient mortality, and lower mortality within 90 days of hospital admission. The evidence for lower 90-day mortality among glucarpidase patients relative to the nonglucarpidase groups is noteworthy, because the study population tended to suffer from AKI, with resultant risk of developing CKD, cardiovascular events, and mortality. ${ }^{33,34} \mathrm{We}$ found no statistically significant association between glucarpidase use and 30-day mortality or 90-day readmission rates. Our findings show that patients treated with glucarpidase had lower LOS and lower inpatient and 90-day mortality than patients who were not treated with glucarpidase.

Our exploratory analysis of the timing of glucarpidase administration showed that patients who received glucarpidase within 3 days of hospital admission spent on average $\sim 8.1$ fewer days in the ICU compared to patients who received glucarpidase later in their hospital stay. They also had shorter overall average LOS in the hospital; however, this difference was partially driven by the longer time to glucarpidase administration that resulted from the definition of the late cohort. These findings are consistent with clinical benefits observed in previous studies, including decreased rates of grade 4 toxicity when glucarpidase is given earlier, as well as with recently developed consensus guidelines to administer glucarpidase within 48-60 hours from starting HDMtx infusion in patients with indication of delayed Mtx clearance in order to minimize life-threatening toxicity., ${ }^{43}$

Our study has several limitations. First, we were limited by Medicare claims data, which do not provide information on Mtx toxicity; therefore, we could not confirm that the nonglucarpidase groups had been diagnosed with Mtx toxicity. Instead, our nonglucarpidase groups consisted of patients who were likely treated for Mtx toxicity based on their diagnosis of PCNSL, for which Mtx is a primary treatment, and indications of inpatient chemotherapy, AKI, and adverse effects/poisoning due to cancer chemotherapy and immunosuppressant drugs. Second, the decision to use glucarpidase is likely to be correlated with illness severity, which may not be observable in Medicare claims data, and may have contributed to the outcome differences between the glucarpidase and nonglucarpidase groups. Third, our samples for the glucarpidase and dialysis ${ }^{+}$groups were small, which may have contributed to the lack of statistical power to detect differences between groups for some outcomes. Furthermore, our study focused on the Medicare population, which consists primarily of elderly people older than 65 years, so our findings may not be generalizable to the broader population of patients with selected cancers. Finally, the exploratory analysis on the early and late users of glucarpidase is presented only for descriptive purposes, and additional research is needed to better understand how the timing of glucarpidase use may be associated with patient outcomes.

\section{Conclusion}

Mtx toxicity is a serious complication with potentially severe impacts on patient outcomes. Medicare cancer patients who receive conventional care for presumed Mtx toxicity experience long hospitalizations, high ICU use, and high mortality. Our findings showed that for Medicare beneficiaries with cancer experiencing Mtx toxicity, treatment with glucarpidase may be associated with shortened LOS, lower inpatient mortality, and lower 90-day mortality. We found no statistically significant relationships between glucarpidase and 90-day readmission rate. To the extent that the lower LOS 
observed among glucarpidase patients was associated with glucarpidase treatment, cost savings from LOS reductions may partially offset glucarpidase costs. These findings are consistent with benefits of glucarpidase on resource utilization and survival, both during hospitalization and beyond. Additional research should examine the effectiveness of glucarpidase on LOS, mortality, and readmissions, and examine the relationship between glucarpidase and additional outcomes, such as cardiovascular events, ESRD, and overall cost to Medicare of treating patients with Mtx toxicity.

\section{Data sharing statement}

The data for this study were acquired from the Centers for Medicare and Medicaid Services. Our data-use agreement (DUA) with CMS precludes us from sharing the data publicly.

\section{Acknowledgments}

The authors would like to thank David Lee, Nikhil Chauhan, Christon Hill, Channeary McDowell, Asha Saavoss, and Sheila Sankaran for their feedback on the data analysis and draft version of this manuscript. This study was funded by BTG International Inc. One author is an employee of BTG International. In addition, other employees/consultants of BTG International reviewed the manuscript and provided comments. BTG International approved the manuscript before submission. The findings from this study were presented as a poster presentation at the Hematology Oncology Pharmacy Association 14th Annual Conference that took place in Denver, CO between March 21, 2018 and March 24, 2018. The poster was presented on March 22, 2018 by Suzanne Ward.

\section{Author contributions}

$\mathrm{BD}, \mathrm{LK}$, and $\mathrm{SW}$ made substantial contributions to study conception and design. BD, LK, JK, CF, EGH, MSA, and SW acquired, analyzed, and interpreted the data, drafted the paper/revised it critically for intellectual content, and gave final approval of the version to be published. All authors agree to be accountable for all aspects of the work.

\section{Disclosure}

This research was sponsored by BTG International, which manufactures the product evaluated in this study. We report that one author (SW) is employed by the company that may be affected by the research reported in the enclosed paper. Four authors (BD, LK, EGH, and MSA) are consultants to BTG International, which may be affected by the research reported in the enclosed paper. One of the authors (JK) served on a speaker panel for glucarpidase for BTG International. BD is an employee of KNG Health Consulting, which received consulting fees from BTG International. LK is an employee of KNG Health Consulting, which received consulting fees from BTG International. CF is a former employee of $\mathrm{KNG}$ Health Consulting, which received consulting fees from BTG International. She is currently affiliated with Boston Scientific, Minneapolis, MN. EGH is an employee of KNG Health Consulting, which received consulting fees from BTG International. MSA is an employee of KNG Health Consulting, which received consulting fees from BTG International. $\mathrm{SW}$ is an employee of BTG International. The authors report no other conflicts of interest in this work.

\section{References}

1. Ward S, King T, Chauhan N. Pooled analysis of time to administration of glucarpidase for methotrexate toxicitiy versus mortality. Clin Toxicol. 2013;51(7):577-578.

2. Flombaum CD, Liu D, Yan SQ, et al. Management of patients with acute methotrexate nephrotoxicity with high-dose leucovorin. Pharmacotherapy. Epub 2018 Jun 4.

3. Buchen S, Ngampolo D, Melton RG, et al. Carboxypeptidase G2 rescue in patients with methotrexate intoxication and renal failure. BrJ Cancer. 2005;92(3):480-487.

4. Widemann BC, Balis FM, Kim A, et al. Glucarpidase, leucovorin, and thymidine for high-dose methotrexate-induced renal dysfunction: clinical and pharmacologic factors affecting outcome. J Clin Oncol. 2010;28(25):3979-3986.

5. Widemann BC, Jayaprakash N, Howard SC, et al. Clinical trial and compassionate use experience with glucarpidase for methotrexate toxicity. J Oncol Pharm Pract. 2014;20(2 SUPPL. 1):8-9.

6. Schwartz S, Borner K, Müller K, et al. Glucarpidase (carboxypeptidase g2) intervention in adult and elderly cancer patients with renal dysfunction and delayed methotrexate elimination after high-dose methotrexate therapy. Oncologist. 2007;12(11):1299-1308.

7. Patterson DM, Lee SM. Glucarpidase following high-dose methotrexate: update on development. Expert Opin Biol Ther. 2010;10(1): $105-111$.

8. Green JM. Glucarpidase to combat toxic levels of methotrexate in patients. Ther Clin Risk Manag. 2012;8:403-413.

9. Widemann BC, Balis FM, Kempf-Bielack B, et al. High-dose methotrexate-induced nephrotoxicity in patients with osteosarcoma. Cancer. 2004;100(10):2222-2232.

10. Fermiano M, Bergsbaken J, Kolesar JM. Glucarpidase for the management of elevated methotrexate levels in patients with impaired renal function. Am J Health Syst Pharm. 2014;71(10):793-798.

11. Cavone JL, Yang D, Wang A. Glucarpidase intervention for delayed methotrexate clearance. Ann Pharmacother. 2014;48(7):897-907.

12. de Miguel D, García-Suárez J, Martín Y, Gil-Fernández JJ, Burgaleta C. Severe acute renal failure following high-dose methotrexate therapy in adults with haematological malignancies: a significant number result from unrecognized co-administration of several drugs. Nephrol Dial Transplant. 2008;23(12):3762-3766.

13. Martinez A, Lebrun J, Clarivet B. Effect of glucarpidase on methotrexate levels and renal function: about five cases. Fundam Clin Pharmacol. 2017;31(Supplement 1):59.

14. Nowicki TS, Bjornard K, Kudlowitz D, Sandoval C, Jayabose S. Early recognition of renal toxicity of high-dose methotrexate therapy. J Pediatr Hematol Oncol. 2008;30(12):950-952.

15. Wyatt KD, Cooper J, Scott K, et al. Delayed methotrexate clearance despite carboxypeptidase-G2 (glucarpidase) administration in 2 patients with toxic methotrexate levels. J Pediatr Hematol Oncol. 2018;40(2):152-155. 
16. Park ES, Han KH, Choi HS, Shin HY, Ahn HS. Carboxypeptidase-G2 rescue in a patient with high dose methotrexate-induced nephrotoxicity. Cancer Res Treat. 2005;37(2):133-135.

17. Vilay AM, Mueller BA, Haines H, Alten JA, Askenazi DJ. Treatment of methotrexate intoxication with various modalities of continuous extracorporeal therapy and glucarpidase. Pharmacotherapy. 2010;30(1):111.

18. Snyder RL. Resumption of high-dose methotrexate after methotrexateinduced nephrotoxicity and carboxypeptidase G2 use. Am J Health Syst Pharm. 2007;64(11):1163-1169.

19. Connors NJ, Sise ME, Nelson LS, Hoffman RS, Smith SW. Methotrexate toxicity treated with continuous venovenous hemofiltration and leucovorin. Clin Toxicol. 2013;51(4):366-367.

20. Gregianin LJ, de Castro Junior CG, Eick R, Brunetto AL. Successful management of acute renal failure after high-dose methotrexate in a patient with relapsed osteosarcoma. Am J Case Rep. 2008;9:93-96.

21. Sharma M, Vuppalli M, Abro Z, Buffington M. 284 Methotrexate nephrotoxicity: is there a case for hemodialysis in the CPDG2 era? Am J Kidney Dis. 2011;57(4):B87.

22. Trifilio S, Ma S, Petrich A. Reduced-dose carboxypeptidase-G2 successfully lowers elevated methotrexate levels in an adult with acute methotrexate-induced renal failure. Clin Adv Hematol Oncol. 2013;11(5):322-323.

23. Ramamoorthy SK, Hephziba R. Acute renal failure post high dose methotrexate infusion successfully managed with high dose folinic Acid and high flux dialysis. Indian J Hematol Blood Transfus. 2013;29(2):90-92.

24. Tuffaha HW, Al Omar S. Glucarpidase rescue in a patient with highdose methotrexate-induced nephrotoxicity. J Oncol Pharm Pract. 2011;17(2):136-140.

25. Boelens AD, Mathôt RAA, Vlaar APJ, Bouman CSC. Glucarpidase treatment for methotrexate intoxication: a case report and review of the literature. Neth J Med. 2018;76(1):36-39.

26. Saland JM, Leavey PJ, Bash RO, Hansch E, Arbus GS, Quigley R. Effective removal of methotrexate by high-flux hemodialysis. Pediatr Nephrol. 2002;17(10):825-829.

27. Harms J, Khawaja A, Taylor M, Han X, Mrug M. Recovery of methotrexate-induced anuric acute kidney injury after glucarpidase therapy. SAGE Open Med Case Rep. 2017;5:2050313X1770505.

28. Grosjean F, Sepe V, Gotti M, Rossi M, dal Canton A. Methotrexate renal clearance by low-dose dopamine in severe nephrotoxicity. Ren Fail. 2016;38(3):362-363.

29. Howard SC, McCormick J, Pui CH, Buddington RK, Harvey RD. Preventing and managing toxicities of high-dose methotrexate. Oncologist. 2016;21(12):1471-1482.

30. Pitman SW, Parker LM, Tattersall MH. Clinical trial of high-dose methotrexate (NSC-740) with citrovorum factor (NSC-3590)-toxicologic and therapeutic observations. Cancer Chemother Rep. 1975;6:43-49.

31. Von Hoff DD, Penta JS, Helman LJ, Slavik M. Incidence of drug-related deaths secondary to high-dose methotrexate and citrovorum factor administration. Cancer Treat Rep. 1977;61(4):745-748.

32. Glezerman IG, Jaimes EA. Chapter 11. Chemotherapy and kidney injury. American Society of Nephrology. New York (NY): Onco-Nephrology; 2016:1-10.

33. Silver SA, Siew ED. Follow-up care in acute kidney injury: lost in transition. Adv Chronic Kidney Dis. 2017;24(4):246-252.

34. Arias-Cabrales C, Rodríguez E, Bermejo S, et al. Short- and long-term outcomes after non-severe acute kidney injury. Clin Exp Nephrol. 2018;22(1):61-67.
35. Park ES, Han KH, Choi HS, Shin HY, Ahn HS. Carboxypeptidase-G2 rescue in a patient with high dose methotrexate-induced nephrotoxicity. Cancer Res Treat. 2005;37(2):133-135.

36. Harms J, Khawaja A, Taylor M, Han X, Mrug M. Recovery of methotrexate-induced anuric acute kidney injury after glucarpidase therapy. SAGE Open Med Case Rep. 2017;5:2050313X1770505.

37. Meyers PA, Flombaum C. High-dose methotrexate-induced renal dysfunction: is glucarpidase necessary for rescue? J Clin Oncol. 2011;29(7):e180.

38. Widemann BC, Adamson PC. Reply to P.A. Meyers et al. J Clin Oncol. 2011;29(7):e181.

39. Wall SM, Johansen MJ, Molony DA, Dubose TD, Jaffe N, Madden T. Effective clearance of methotrexate using high-flux hemodialysis membranes. Am J Kidney Dis. 1996;28(6):846-854.

40. Voraxaze. FDA Label. (n.d.): 2012. Available from: https://www.accessdata.fda.gov/drugsatfda_docs/label/2012/125327lbl.pdf. Accessed January 3, 2019

41. Widemann BC, Schwartz S, Jayaprakash N, et al. Efficacy of glucarpidase (carboxypeptidase g2) in patients with acute kidney injury after high-dose methotrexate therapy. Pharmacotherapy. 2014;34(5):427-439.

42. Widemann BC, Adamson PC. Understanding and managing methotrexate nephrotoxicity. Oncologist. 2006;11(6):694-703.

43. Ramsey L, Balis FM, O’Brien MM. Consensus guidelines for the use of glucarpidase in patients with high-dose methotrexate induced acute kidney injury and delayed methotrexate clearance. Oncologist. 2018;23(1):52-61.

44. Grommes C, DeAngelis LM. Primary CNS lymphoma. J Clin Oncol. 2017;35(21):2410-2418.

45. Rubenstein JL, Gupta NK, Mannis GN, Lamarre AK, Treseler P. How I treat CNS lymphomas. Blood. 2013;122(14):2318-2330.

46. Blay JY, Conroy T, Chevreau C, et al. High-dose methotrexate for the treatment of primary cerebral lymphomas: analysis of survival and late neurologic toxicity in a retrospective series. J Clin Oncol. 1998;16(3):864-871.

47. Yale New Haven Health Services Corporation/Center for Outcomes Research \& Evaluation (YNHHSC/CORE). "2015 Measure Updates and Specifications Report: Hospital-Wide All-Cause Unplanned Readmission Measure - Version 4.0"; 2015b. Available from: https://www. qualitynet.org/dcs/ContentServer?c=Page\&pagename=QnetPublic\%2 FPage\%2FQnetTier3\&cid=1228774371008. Accessed July 1, 2016.

48. Elixhauser A, Steiner C, Harris DR, Coffey RM. Comorbidity measures for use with administrative data. Med Care. 1998;36(1):8-27.

49. Austin PC. An introduction to propensity score methods for reducing the effects of confounding in observational studies. Multivariate Behav Res. 2011;46(3):399-424.

50. Austin PC, Stuart EA. Moving towards best practice when using inverse probability of treatment weighting (IPTW) using the propensity score to estimate causal treatment effects in observational studies. Stat Med. 2015;34(28):3661-3679.

51. Stuart EA, Lee BK, Leacy FP. Prognostic score-based balance measures can be a useful diagnostic for propensity score methods in comparative effectiveness research. J Clin Epidemiol. 2013;66(8 Supp1): S84-S90.e1.

52. Garrido MM, Kelley AS, Paris J, et al. Methods for constructing and assessing propensity scores. Health Serv Res. 2014;49(5): 1701-1720. 


\section{Supplementary material}

Table SI Variable definitions

\begin{tabular}{|c|c|c|c|c|}
\hline & $\begin{array}{l}\text { ICD9 diagnosis } \\
\text { codes }\end{array}$ & $\begin{array}{l}\text { ICD I } 0 \text { diagnosis } \\
\text { codes }\end{array}$ & $\begin{array}{l}\text { ICD9 procedure } \\
\text { codes }\end{array}$ & ICDIO procedure codes \\
\hline Glucarpidase & & & 0.95 & 3E033GQ, 3E043GQ \\
\hline $\begin{array}{l}\text { Adverse effects, } \\
\text { poisoning by } \\
\text { antineoplastic and } \\
\text { immunosuppressive } \\
\text { drugs }\end{array}$ & E933.I, 963.I & $\begin{array}{l}\text { T45IX5A, T45IX5S, } \\
\text { T45IXIA, T45IX2A, } \\
\text { T45IX3A, T45IX4A }\end{array}$ & - & - \\
\hline $\begin{array}{l}\text { Inpatient } \\
\text { chemotherapy }\end{array}$ & $\begin{array}{l}\text { V58.IX, V662, } \\
\text { V672 }\end{array}$ & Z5I0, Z5IIII, Z5III2 & $\begin{array}{l}99.28,99.25 \\
17.70,00.10 \\
00.15\end{array}$ & 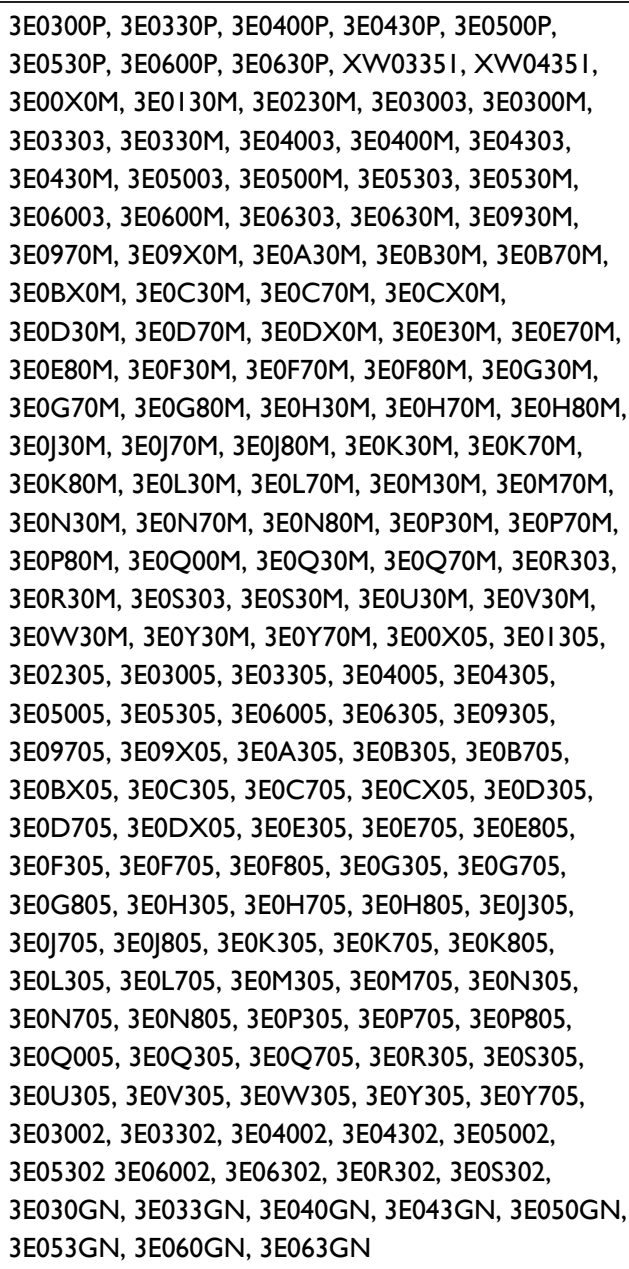 \\
\hline \multirow{2}{*}{$\begin{array}{l}\text { Leukemia/lymphoma } \\
\text { and other malignant } \\
\text { lymphomas }\end{array}$} & $200 *, 204 . *$ & $\mathrm{C} 83^{* *}, \mathrm{C} 9 I^{* *}$ & - & - \\
\hline & 202.8 & C8580, C8589 & - & - \\
\hline $\begin{array}{l}\text { Primary central } \\
\text { nervous system } \\
\text { lymphoma }\end{array}$ & $\begin{array}{l}200.30,200.50 \\
200.51,200.52 \\
200.53,200.54 \\
200.55,200.56 \\
200.57,200.58 \\
200.80,202.80\end{array}$ & $\begin{array}{l}\text { C83.89, C83.39, } \\
\text { C85.89 }\end{array}$ & & \\
\hline Acute renal injury & $584 .^{*}$ & $\begin{array}{l}\text { NI70, NI7I, NI72, } \\
\text { NI78, NI79, O904 }\end{array}$ & - & - \\
\hline
\end{tabular}

(Continued) 
Table SI (Continued)

\begin{tabular}{|c|c|c|c|c|}
\hline & $\begin{array}{l}\text { ICD9 diagnosis } \\
\text { codes }\end{array}$ & $\begin{array}{l}\text { ICD I } 0 \text { diagnosis } \\
\text { codes }\end{array}$ & $\begin{array}{l}\text { ICD9 procedure } \\
\text { codes }\end{array}$ & ICDIO procedure codes \\
\hline \multirow[t]{3}{*}{$\begin{array}{l}\text { Acute or chronic renal } \\
\text { injury }\end{array}$} & $584 .^{*}$ & $\begin{array}{l}\text { NI70, NI7I, NI72, } \\
\text { NI78, NI79, O904 }\end{array}$ & - & - \\
\hline & $\begin{array}{l}\text { 585.*, 586.*, V56.*, } \\
\text { 588.8*, V420.*, } \\
\text { V45I.*, 404I2, } \\
\text { 404I3, 40492, } \\
\text { 40493, 4030I, } \\
\text { 403II, 4039I, } \\
\text { 40402, 40403 }\end{array}$ & $\begin{array}{l}\text { II 20*, II } 3 I^{*}, \text { N250*, } \\
\text { Z490*, Z49I*, Z492*, } \\
\text { Z940*, Z992*, N18*, } \\
\text { NI9* }\end{array}$ & - & - \\
\hline & $593 .^{*}$ & $\begin{array}{l}\text { N2883, N288I, } \\
\text { N28I, NI35, NI38, } \\
\text { NI34, R802, NI370, } \\
\text { NI37I, NI372I, } \\
\text { NI3722, NI3729, } \\
\text { N280, N2882, } \\
\text { N2889, N289 }\end{array}$ & - & - \\
\hline
\end{tabular}

\section{Literature search strategy}

We conducted a literature review to identify published articles that reported patient outcomes associated with the use of glucarpidase in alleviating methotrexate (Mtx) toxicity in cancer patients. Patient outcomes examined included length of stay, readmissions, and mortality. A systematic literature review was performed using Embase (Wolters Kluwer Health, New York, NY, USA) and PubMed (National Center for Biotechnology Information, National Institutes of Health, Bethesda, MD, USA) databases for the following search terms: glucarpidase, methotrexate, toxicity, poisoning, cancer, mortality, readmissions, and length of stay (see below for the complete list of search terms and search queries). An initial screening for articles published in English between January 2000 and October 2018 was performed.

Thereafter, titles, abstracts, and full texts were screened to limit the studies to those focused on cancer patients being treated for Mtx and/or nephrotoxicity. Several studies examined clinical outcomes, such as serum creatinine levels and other renal function indicators, but did not report patient outcomes of interest in this literature review (ie, readmissions, mortality, length of stay). These studies were excluded from the literature review. An Excel worksheet (Microsoft, Redmond, WA, USA) was created to compile all relevant data. To ensure a comprehensive search, references within identified studies were further searched to include relevant articles missed in the initial search, and clinical experts reviewed our literature review to ensure we included all significant literature on the topic of Mtx toxicity and glucarpidase rescue. After title, abstract, and full-text screening, a total of 28 articles met all the inclusion/exclusion criteria and were included in the review (see below for literature review attrition chart).

\section{Search terms}

We used controlled vocabulary to identify articles on PubMed and Embase. We used the following terms to create combinations of keywords to identify studies:

- glucarpidase

- carboxypeptidase $\mathrm{G}_{2}$

- methotrexate

- length of stay

- readmissions

- intensive care unit

- mortality

- toxicity

- poison

- acute kidney injury

- nephrotoxicity 


\section{Search queries}

(((((length of stay OR readmissions OR intensive care unit OR mortality)) AND (toxicity or poison))) AND glucarpidase) AND methotrexate ("toxicity”/exp OR toxicity OR “intoxication"/exp OR intoxication OR “poisoning”/exp OR poisoning) AND ("carboxypeptidase g2"/exp OR "carboxypeptidase g2" OR "glucarpidase"/exp OR glucarpidase) AND ("methotrexate"/exp OR methotrexate) AND (("length of stay"/exp OR "length of stay" OR "hospital readmission"/exp OR "hospital readmission" OR "mortality"/exp OR mortality) OR ("intensive care unit"/exp OR "intensive care unit")) AND [english]/lim AND [2000-2018]/py

((“carboxypeptidase G2”/exp OR “carboxypeptidase g2") OR ("glucarpidase”/exp OR glucarpidase)) AND (“methotrexate”/exp OR methotrexate) AND (("acute kidney failure"/exp OR "acute kidney failure") OR ("acute kidney injury"/exp OR "acute kidney injury" OR (acute AND ("kidney"/ exp OR kidney) AND ("injury"/exp OR injury))) OR (“nephrotoxicity”/exp OR nephrotoxicity)) AND [english]/lim AND [2000-2018]/Py

((((carboxypeptidase-G2) OR glucarpidase)) AND methotrexate) AND ((acute kidney injury) OR nephrotoxicity) Filters: publication date from January I, 2000; English 


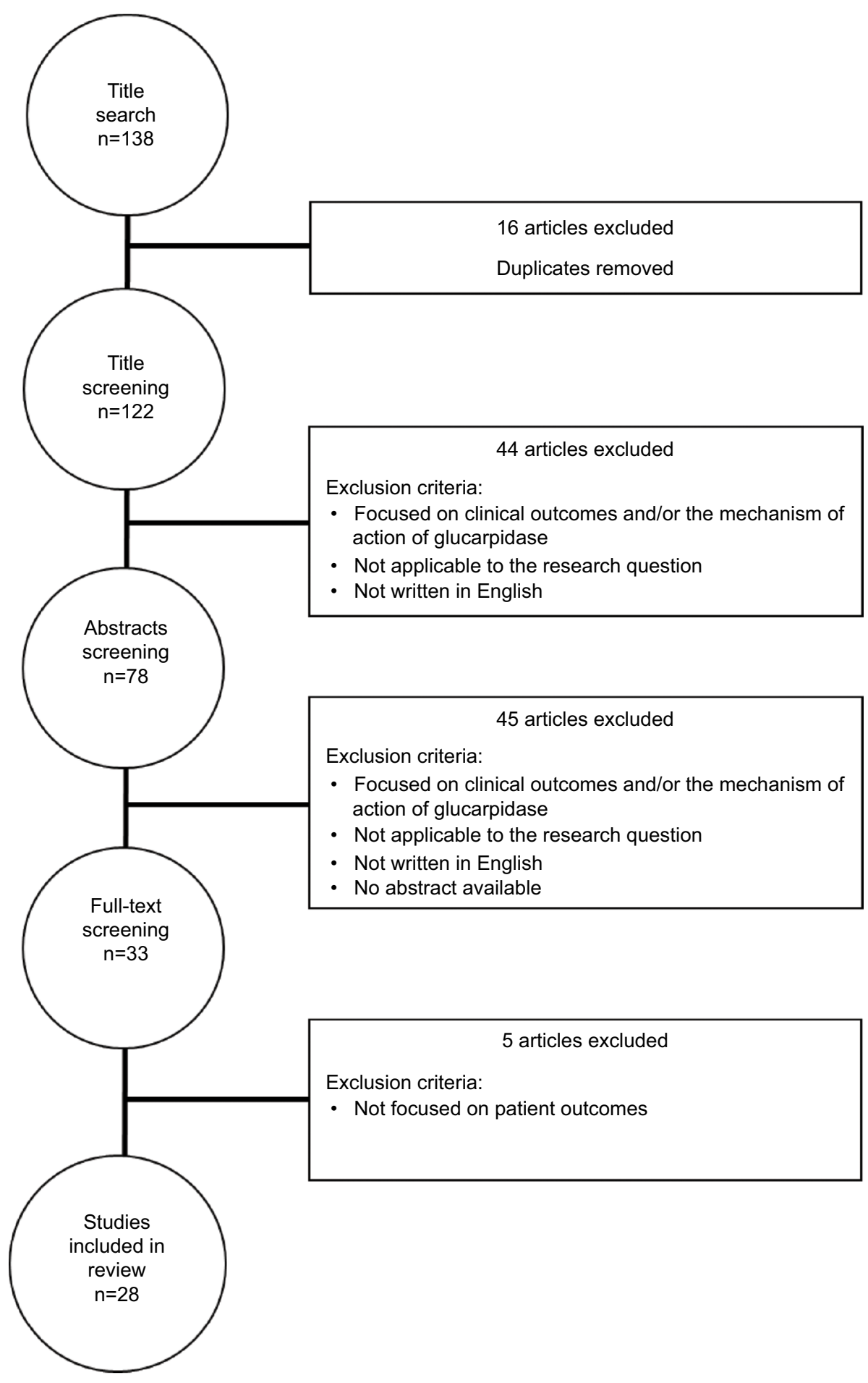

Figure SI Attrition chart.

ClinicoEconomics and Outcomes Research

\section{Publish your work in this journal}

ClinicoEconomics and Outcomes Research is an international, peerreviewed open-access journal focusing on health technology assessment, pharmacoeconomics and outcomes research in the areas of diagnosis, medical devices, and clinical, surgical and pharmacological intervention. The economic impact of health policy and health systems
Dovepress

organization also constitute important areas of coverage. The manuscript management system is completely online and includes a very quick and fair peer-review system, which is all easy to use. Visit http://www.dovepress.com/testimonials.php to read real quotes from published authors.

Submit your manuscript here: https://www.dovepress.com/clinicoeconomics-and-outcomes-research-journal 